SANJA MARUŠIĆ, Ph.D.

University of Zagreb,

Faculty of Transport and Traffic Sciences

Vukelićeva 4, HR-10000 Zagreb, Republic of Croatia
Science in Traffic and Transport Original Scientific Paper

Accepted: Jan. 20, 2009

Approved: Oct. .22, 2009

\title{
A NOTE ON BOUNDARY CONDITIONS FOR THE LWR MODEL
}

\section{ABSTRACT}

The paper studies the boundary conditions for the standard $L W R$ model describing the traffic flow. The notion of the BLN (Bardos, Leroux and Nédélec) condition is described. In the context of traffic flow the BLN conditions have some natural interpretation. The conditions on the density and on the flow and their meaning in real-life situations are discussed.

\section{KEY WORDS}

LWR model, traffic flow, hyperbolic conservation law, boundary conditions

\section{INTRODUCTION}

In 1955 in their celebrated paper [9] Lighthill and Witham derived a simple hyperbolic conservation law for the traffic flow based on vehicle conservation principle. The same model was found independently by Richards [11]. In recognition of their pioneering work such model is called the LWR model.

The fundamental assumption of all continuum models, including the LWR model, is that the density function $\rho$ can be properly defined. Indeed, the continuum models for traffic flows do not allow individual tracking of cars, but they describe the dynamics of the macroscopic density of cars. Therefore, the unknown quantity in this problem is the car density $\rho$ roughly defined as the number of vehicles per unit of length [3]. Another assumption characteristic for the LWR model is that the speed $v$ is a function depending only on the density, i. e. that

$v=V(\rho)$

Such function $V$ is empirical and it depends on the characteristics of the road. Several different models have been proposed by taking different functions $V(\rho)$. The review of such models and their properties can be found, for instance, in [10] and [6]. It is commonly assumed in the traffic flow analysis that the car flow $q(\rho)=\rho V(\rho)$ satisfies $q(0)=q\left(\rho_{\max }\right)=0$ and that $q$ attains its maximum $q_{\max }=q\left(\rho_{c}\right)$ (= road capacity), for some optimal density $\rho_{c} \in\left(0, \rho_{\max }\right)$. The usual empirical curve for flow versus density is similar to the one in Figure 1 (so-called fundamental diagram of road traffic). Such curve is obviously strictly concave.

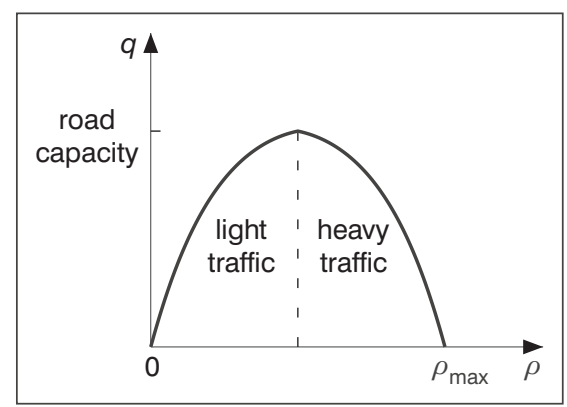

Figure 1 - Traffic flow curve

The exception is the so called California model, where the function $\rho \longmapsto q(\rho)$ is linear

$q(\rho)=v_{0}\left(1-\frac{\rho}{\rho_{\max }}\right)$

Using the simple car conservation principle, analogous to the mass conservation in continuum mechanics, leads to the local conservation law

$\frac{\partial \rho}{\partial t}+\frac{\partial q}{\partial x}=0$

Since $q "<0$ (preserves the sign) that places the law (3) in the class of hyperbolic conservation laws. If some initial density $\rho_{0}$ at time $t=0$ is prescribed the Cauchy's problem for hyperbolic partial differential equation (PDE) of the first order is obtained:

$\frac{\partial \rho}{\partial t}+\frac{\partial q(\rho)}{\partial x}=0, x \in \mathbf{R}, t>0$

$\rho(x, 0)=\rho_{0}(x), x \in \mathbf{R}$.

To be able to pick the unique physically relevant solution an entropy condition is added (see e. g. [7] and [1]). Most of the theoretical papers on LWR model are limited to the study of such Cauchy's problems, i. e. they assume that the road has no ends. However, in real-life situations, the road is always bounded, i. e. it has an entry and an exit. Furthermore, for the purpose of numerical simulations it is necessary to restrict on a bounded part of the road limited by two-point boundary. For such a problem the initial condition is, in general, not sufficient to assure the 
well-posedness and some boundary conditions are needed. For the purpose of numerical simulations, often, the non physical (artificial) conditions, like periodicity of the density, are imposed. The first physically reasonable boundary conditions that we can think of are the Dirichlet's conditions. It seems natural to prescribe the car density at the entry and at the exit of the space interval (since there is one-to-one relationship between the density and the speed we could equivalently prescribe the speed).

Remark 1: Actually, as we know, the hyperbolic conservation laws, in general, do not admit smooth or even continuous solutions so the question whether the value of such function on the boundary has a sense is nontrivial. Even though for smooth data such solution is peacewise smooth, the trace on the boundary cannot simply be taken as the value of the solution on a boundary. If, for example, there are two constants $a$ and $\rho_{0}$ such that $a<\rho_{c}$ and $\rho_{c}<\rho_{0}<\rho_{\text {max }}$, then the problem

$$
\begin{gathered}
\frac{\partial \rho}{\partial t}+\frac{\partial q(\rho)}{\partial x}=0, x \in \mathbf{R}, t>0 \\
\rho(x, 0)=\rho_{0}, x \in \mathbf{R}, \rho(0, t)=a, \mathrm{t}>0
\end{gathered}
$$

has a unique entropy solution depicted here:

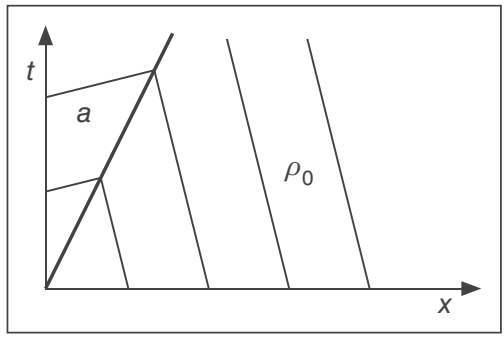

Figure 2 - Entropy solution

Thus

$a=\rho(0,0+) \neq \rho(0+, 0)=\rho_{0}$

where

$$
f(0+)=\lim _{s \rightarrow 0, s>0} f(s)=\lim _{s \rightarrow 0+} f(s)
$$

denotes the right-hand side limit of function fin point 0. Although hyperbolic conservation laws do not have continuous solutions, their solutions have bounded the total variation. This property allows to consider the limit of $\rho(x, t)$ as $x \rightarrow 0+$ in some technical sense (more precisely, in $L^{1}$ sense). This limit is called the trace of $\rho(x, t)$ on boundary $x=0$. Furthermore, such trace is a measurable function bounded almost everywhere on $] 0, T[$ and its value on the boundary $x=0$ can be imposed:

$$
\rho(0, t)=a(t) \text { for almost every } t \in] 0, T[
$$

Thus, the value of trace $\rho(0, t)$, defined in such a way, in one point $t=0$ has no meaning. For technical details the reader should consult [2]. The same discussion holds for the right-hand boundary $x=l$.

In the above example, the data do not verify the compatibility condition $\rho_{0}(0)=a(0)$ (usually imposed for the parabolic problems) and the trace discontinuity (6) is hardly surprising.

In the traffic flow theory such problems are also called the three detector problems (see [3]). The space interval is denoted by $I=[0, l]$, the value of is $\rho$ that is to be imposed at point $x=0$ by $a(t)$, and the value of $\rho$ for $x=0$ by $b(t)$. The Dirichiet conditions are to be imposed

$\rho(0, t)=a(t)$

$\rho(l, t)=b(t)$

and due to Remark 1 , that traces $\rho(0, t)$ and $\rho(0, l)$ are meaningful, even for weak solutions. Nevertheless, things are not so simple. Obviously, the value of $\rho$ at the entry of interval $x=0$ cannot be prescribed if the characteristics have a negative slope (i. e. when $q^{\prime}<$ 0 ), otherwise they contradict the initial condition.

For the same reason we cannot prescribe the density at the exit $x=l$ when the characteristics have a positive slope (i. e. when $q$ ' $>0$ ). It is less obvious that, even if the slope of characteristics at the entry is positive, we are not sure that the condition (7) could be imposed without violating the entropy condition.

The condition that guarantees the well-posedness (i. e. the existence of a unique entropy solution) for the boundary value problem (4), (5), (7) and (8) is a kind of entropy condition on the boundary that can be formulated as follows:

$$
\begin{aligned}
& \frac{q(\rho(0, t))-q(k)}{\rho(0, t)-k} \leq 0 \\
& \quad \text { for every } k \text { between } \rho(0, t) \text { and } a(t) \\
& \frac{q(\rho(l, t))-q(k)}{\rho(l, t)-k} \geq 0 \\
& \quad \text { for every } k \text { between } \rho(l, t) \text { and } b(t)
\end{aligned}
$$

The geometric interpretation of the above conditions is that the slope of the cord joining $(\rho(0, t), q(\rho(0, \tau)))$ and $(k, q(k))$, for any $k$ between $\rho(0, t)$ and $a(t)$, is negative, while the slope of the cord between $(\rho(l, t), q(\rho(l, \tau)))$ and $(k, q(k))$, for any $k$ between $\rho(l, t)$ and $b(t)$, is positive.

In the mathematical literature such conditions are known as the BLN conditions, named after Bardos, Leroux and Nédélec.

If (9) and (10) hold, we say that $\rho$ satisfies the Dirichiet's boundary conditions (7), (8) in the BLN sense.

For the rigorous mathematical derivation of BLN boundary conditions in general context one can consult [2] or [12]. It should be mentioned that the BLN condition does not work for hyperbolic systems, i. e. for the second order models of traffic flow. Such situation is more complex and was considered by Dubois and Le Floche in [4] using the Riemann problem method. In case of scalar equation, their boundary conditions are equivalent to the BLN conditions. Another technique based on the asymptotic analysis of a 
solution to the appropriate viscous system in a boundary layer, similar to the original method of Bardos, Leroux and Nédélec was used in [5] and [12].

In the present paper the BLN condition in the specific context of the LWR model for a traffic flow is analyzed and given a meaning.

Remark 2: Before completing this section it should be stressed that the traces $\rho(0, t)$ and $\rho(l, t)$ are to be understood as $\rho(0+, t)$ and $\rho(l-, t)$ respectively. On the other hand, $a(t)$ and $b(t)$ can, in some loose sense, be seen as $\rho(0-, t)$ and $\rho(l+, t)$.

\section{ANALYSIS OF BLN CONDITIONS IN CASE OF LWR MODEL}

The meaning of such boundary conditions written in the form of variation inequalities (9) and (10) is unclear. A simple analysis allows their notation in a different way that will help understand their meaning in a real-life situation.

First, left-hand boundary $x=0$ is dealt with:

(i) Suppose that the boundary value $a(t) \leq \rho_{c}$. If $\rho(0, t)<a(t)$ then the function $q$ increases on

$$
[\rho(0, t), a(t)] \text { and } \frac{q(\rho(0, t))-q(k)}{\rho(0, t)-k}<0
$$

for $k \in[\rho(0, t), a(t)]$. Thus (9) is violated meaning that necessarily $\rho(0, t) \geq a(t)$. Let the number $a^{*}(t) \geq \rho_{c}$ be denoted by $a^{*}(t)$ such that $q(a(t))=q\left(a^{*}(t)\right)$.

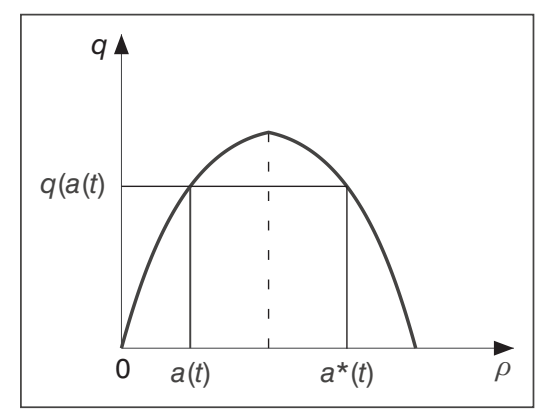

Figure 3 - Values $a(t)$ and $a^{*}(t)$

Now, if $a(t)<\rho(0, t)<a^{*}(t)$, then $q(k) \leq q(\rho(0, t))$ for any $k \in[a(t), \rho(0, t)]$ and (9) is again violated. Finally, if $\rho(0, t) \geq a^{*}(t)$ then $q(k) \geq q(\rho(0, t))$ for any $k \in[a(t), \rho(0, t)]$ so that (9) holds. Thus the only two possibilities are:

either $\rho(0, t)=a(t)$ or $\rho(0, t) \geq a^{*}(t)$

This condition can be equivalently written as:

either $\rho(0, t)=a(t)$ or $\left\{\begin{array}{l}\rho(0, t)>\rho_{c} \\ \text { and } \\ \mathrm{q}(\rho(0, t)) \leq q(a(t))\end{array}\right.$

(ii) The second case is $a(t)>\rho_{c}$. If $\rho(0, t)>a(t)$ function $q$ decreases on $[\rho(0, t), a(t)]$ and (9) is auto- matically fulfilled. If $\rho(0, t) \leq a(t)$ the condition (9) is fulfilled as long as $\rho(0, t) \geq \rho_{c}$ and it does not hold otherwise. Thus the BLN condition (9) is reduced to

$$
\rho(0, t) \geq \rho_{c}
$$

Such condition has nothing to do with the actual value of $a(t)$.

Thus, on the right-hand boundary $x=0$, the BLN boundary condition (9) is reduced to conditions (12) and (13).

In case of the right-hand boundary $x=l$, arguing in the same way the following form of the BLN boundary conditions is obtained:

(i) If $b(t) \geq \rho_{c}$ then the BLN boundary condition can be written in the form

$$
\text { either } \rho(l, t)=b(t) \text { or } \rho(l, t) \leq b^{*}(t),
$$

with $b^{*}(t)<\rho_{c}$ chosen such that $q(b(t))=$ $=q\left(b^{*}(t)\right)$, or equivalently

$$
\text { either } \rho(l, t)=b(t) \text { or }\left\{\begin{array}{l}
\rho(l, t)<\rho_{c} \\
\text { and } \\
\mathrm{q}(\rho(l, t)) \leq q(b(t))
\end{array}\right. \text {. }
$$

(ii) In case $b(t)<\rho_{c}$ we can only impose:

$$
\rho(0, t) \leq \rho_{c} \text {. }
$$

A closer look at (9) shows that the entering density can be (eventually) prescribed only if the value that is to be imposed is smaller than or equal to optimal density $\rho_{c}$ i. e. if the corresponding traffic is light. On the other hand (10) says that the exiting density could be prescribed only if it is larger than or equal to $\rho_{c}$, i. e. if the traffic is heavy. More precisely, it can only be said that the entering density either takes some prescribed value $\rho(0, t)=a(t) \leq \rho_{c}$ or it is high $\left(>\rho_{c}\right)$ and the corresponding flow $q(\rho(0, t))$ is smaller than $q(a(t))$, but we can neither prescribe its exact high value nor can we say for which $t$ it is assumed (without solving the mixed problem (4), (5), (9) and (10)). A similar discussion holds for the exiting density.

We can also have the one-point boundary. Such problem is called a semi-infinite highway problem (see [3], [6]). The space domain is $[0, \infty\rangle$ and the value of the density at the entry $x=0$ is prescribed. The same problem as before appears and such value can be prescribed only in the BLN sense, i. e. (9) can be imposed. So, the entry density can be (eventually) prescribed only if it is suboptimal (i. e. $\leq \rho_{c}$ ).

Finally, we can prescribe the value of flow $q$ on the boundary. In the case of two-point boundary value problem, it can be done but it necessarily creates a shock wave. In case of the semi-infinite highway problem such problem is also well-posed. It also has a clear physical sense because it is natural to prescribe the cumulative flow wishing to enter upstream. 


\section{ANALYSIS OF CHARACTERISTICS}

The purpose of this section is to give some simple arguments in favor of the BLN boundary conditions and to illustrate them by a typical example.

The characteristic for equation (4) is the line in $(x$, $t)$ plane on which the solution retains a constant value. Its slope is given by $1 / q^{\prime}(\rho)$. If a characteristic passes through a point $(x, 0)$ then the value of the solution on it equals $\rho_{0}(x)$. If the characteristic intersects with line $x=0$ then the above argument contradicts the boundary condition $\rho(0, t)=a(t)$, unless $a(t)=\rho_{0}(x)$, which is unlikely.

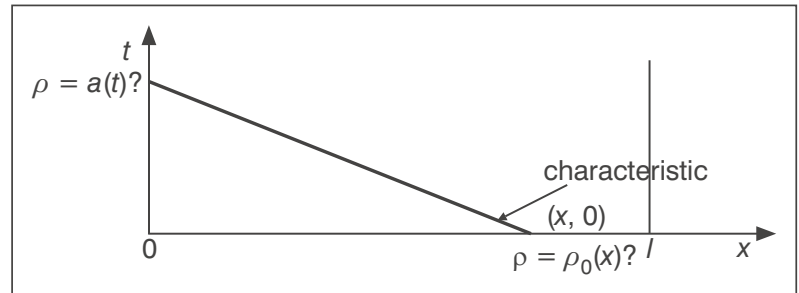

Figure 4 - Characteristic with negative slope

In case the characteristics have a negative slope (i. e. $q^{\prime}<0$ ) at point $x=0$ the boundary condition (7) cannot be imposed. This is in accordance with the BLN condition (12).

The same argument shows that the boundary condition (8) cannot be imposed if the characteristics at point $x=l$ have a positive slope (i. e. $q^{\prime}>0$ ). This corresponds to the right BLN condition (14).

Example 1: Suppose that there is a green traffic light at the entry and that the cars are moving at a maximal flow (i. e. the road capacity). On the exit, there is a red traffic light, i. e. the density is maximal. Then the following is imposed

$$
\begin{array}{r}
\rho(0, t)=\rho_{c}, \rho(l, t)=\rho_{\max } \text { and } \\
\rho(x, 0)=\left\{\begin{array}{l}
\rho_{c} \text { for } 0<x<l / 2 \\
\rho_{\max } \text { for } l / 2 \leq x<l
\end{array} .\right.
\end{array}
$$

It may be noticed that the given data are continuous in the sense $\rho_{0}(0+)=a(0+)$. However, such a situation will necessarily create shock wave propagating at negative speed

$$
\frac{q\left(\rho_{c}\right)}{\rho_{c}-\rho_{\max }} .
$$

This shock wave will reach the entry at time

$$
t^{*}=\frac{\rho_{\max }-\rho_{c}}{2 q\left(\rho_{c}\right)} l .
$$

Thus, the left boundary value of $\rho$ can be prescribed only before the shock reaches the boundary, i. e.

$$
\rho(0, t)=\rho_{c}, t<t^{*} .
$$

After the shock hits the boundary, $\rho$ takes the high value $\rho_{\max }$ which is the second alternative from the BLN condition (12). On the other hand, at the exit there is the Dirichiet's condition

$$
\rho(l, t)=\rho_{\max }, t>0
$$

as long as there is red light at the exit. Of course this feature has a clear explanation. If the exit is closed long enough and there is a constant inflow, this will eventually lead to a congested road with bumper-to-bumper density along its entire length.

One can decide to change the entering traffic light to red after some interval $\tau>0$, i. e.

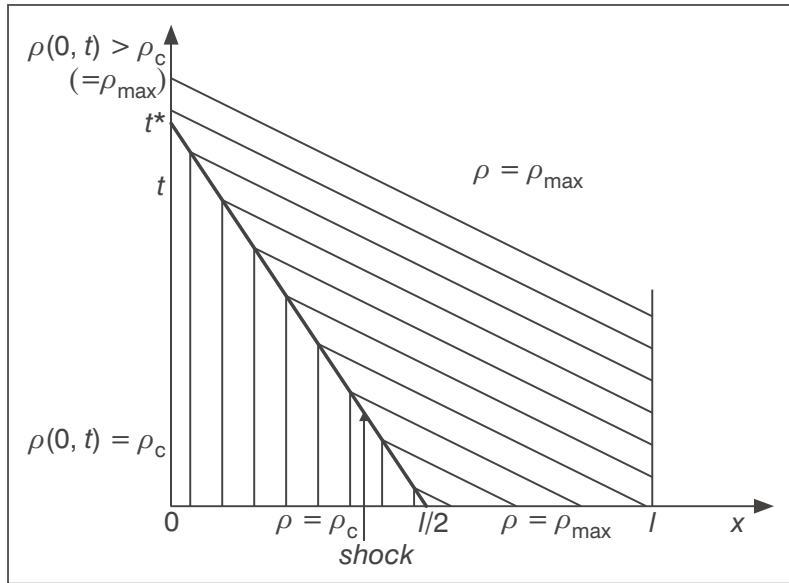

Figure 5 - Simple shock wave

If $\tau>t^{*}$ it would be pointless, because the road is already congested and there is still $\rho(0, t)=\rho_{\max }$.

If $\tau>t^{*}$, then $\rho(0, t)=a(t)$ can be imposed which, after time

$$
t_{0}=\frac{\rho_{\max }-\rho_{c}}{\rho_{\max }}\left(\frac{l \rho_{c}}{2 q\left(\rho_{c}\right)}+\tau\right),
$$

leads to the polarized situation

$$
\rho(x, t)=\left\{\begin{array}{l}
0 \text { for } x<\frac{\rho_{\max }-\rho_{c}}{\rho_{\max }} \frac{l}{2}-\frac{q\left(\rho_{c}\right)}{\rho_{\max }} \tau \\
\rho_{\max } \text { otherwise }
\end{array} .\right.
$$

There is the stationary shock at

$$
x=x_{0}=\frac{\rho_{\max }-\rho_{c}}{\rho_{\max }} \frac{l}{2}-\frac{q\left(\rho_{c}\right)}{\rho_{\max }} \tau
$$

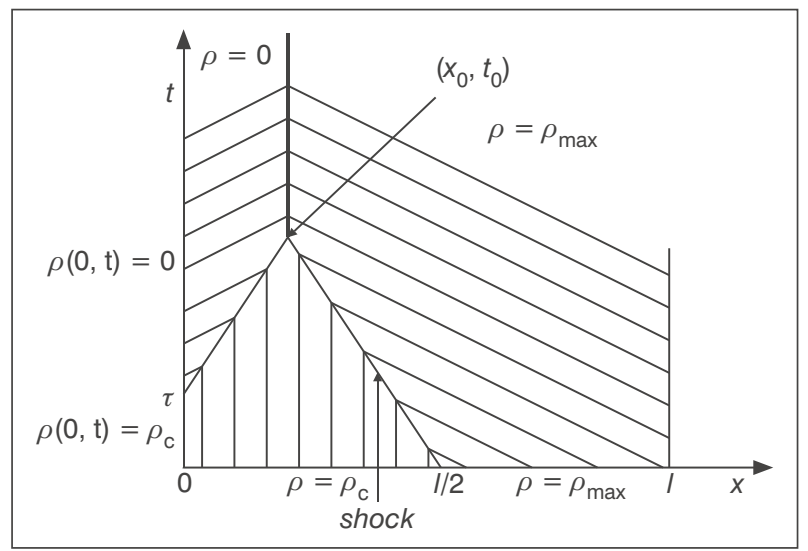

Figure 6 - More complicated example of shock 
which does not touch the boundary $x=0$ and the Dirichiet 's condition remains valid. This situation is in accordance with expectations. All the cars that entered the part of the road $\langle 0, l\rangle$, before the traffic light at the entry turns red at time $\tau$, went for the exit and they are waiting for the exit traffic light to turn green. They occupy the part $\left\langle x_{0}, l\right\rangle$ while the part $\left\langle 0, x_{0}\right\rangle$ remains void. The time when such polarized situation occurs can be computed and equals $t_{0}$.

\section{COMPATIBILITY BETWEEN THE INITIAL AND THE BOUNDARY CONDITION}

For this purpose the Riemann problem approach of Dubois and Le Floche [4] is briefly described.

The focus is on the left boundary and the Riemann problem is considered.

$\frac{\partial u}{\partial t}+\frac{\partial q(u)}{\partial x}=0, x \in \mathbf{R}, t>0$

$u(x, 0)=\left\{\begin{array}{l}a \text { for } x<0 \\ \rho_{0} \text { for } x>0\end{array}, x \in \mathbf{R}\right.$

The solution of such problem depends only on variable $\mathrm{x} / \mathrm{t}$ and it is composed of two constant states separated by an elementary wave. In this case of scalar conservation law with strictly concave nonlinearity, the only possible elementary waves are shock and rarefaction waves. That solution is usually denoted as $u=w\left(x / t, a, \rho_{0}\right)$. From the beginning it is supposed that $a<\rho_{c}$. Again, $a^{*}>\rho_{c}$ is defined such that $q(a)=$ $q\left(a^{*}\right)$.

If $\rho_{0}>a^{*}$ does not develop shock wave in the right quadrant $x>0$ but in the left one $x<0$. Thus, in the right quadrant the solution equals $\rho_{0}$ and it is completely determined by the initial condition $\rho_{0}$ while a has no impact on it. It should be stressed that the boundary value equals $\rho(0, t)=\rho_{0}>a^{*}$.

On the other hand, if $\rho_{0}<a^{*}$ then the solution has, either a shock (in case $a<\rho_{0}<\rho_{c}$ ) or a rarefaction wave (in case $\rho_{0}<a<\rho_{c}$ ) in the right quadrant. Thus, the solution $u(x, t)$ takes both values $a$ as well as $\rho_{0}$ in the right quadrant. In either case the boundary condition $u(0, t)=a$ is fulfilled.

The above example gives us some hint about the meaning of two alternatives in the BLN condition (12). If we neglect the left quadrant and take only the right one, then $u(x, t)$ is an entropy solution of equation (16) with initial condition

$$
u(x, 0)=\rho_{0}
$$

and the boundary condition

$u(0, t)=a$

understood in the BLN sense. Thus, if $\rho_{0}<a^{*}$, then the boundary condition (18) is satisfied in the usual way (i. e. the first alternative from (12) is valid). On the other hand, if $\rho_{0} \geq a^{*}$ then we only have $(0, t) \geq a^{*}$ (indeed, since $\left.u(0, t)=\rho_{0}\right)$, i. e. the second alternative from (12) takes place. So, if we want to impose the density $a$ on the boundary, literary and not in the BLN sense, the initial density $\rho_{0}$ must not be larger than $a^{*}$. By the way, the above example shows that the values $a>\rho_{c}$ would give the characteristics pointing to the left quadrant, i. e. with no effect on the solution in the right quadrant. This explains why such boundary values are not admissible.

Remark 3: As can be seen from the above discussion, the $B L N$ condition (9) can be equivalently written as

$$
\rho(0, t) \in v(a(t))=\left\{w(0+, a(t), k) ; 0 \leq k \leq \rho_{\max }\right\} .
$$

This result is due to Dubois and Le Floche [4], where its rigorous proof can be found, and more general results for the hyperbolic systems.

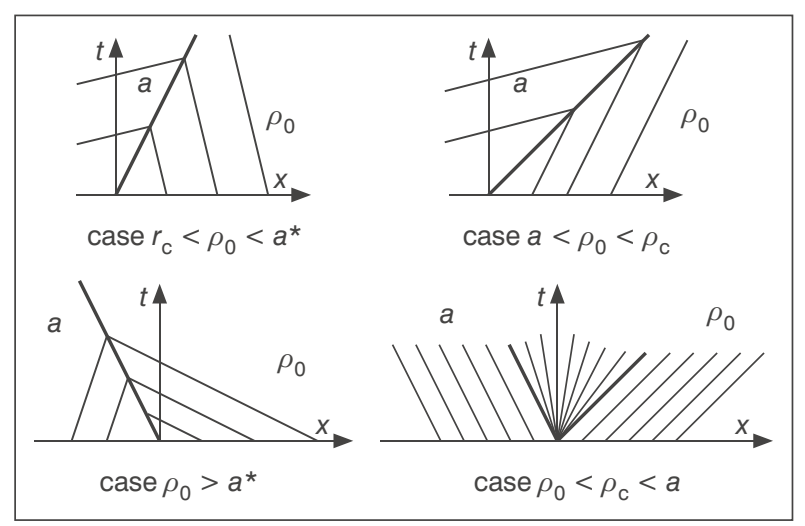

Figure 7 -Three examples of shocks and a rarefaction wave

Let us now go back to the original problem. Applying locally the above analysis at some vicinity of the origin, the objective is to find the compatibility condition that $a(t)$ and $\rho_{0}(x)$ need to satisfy if the boundary condition is to be imposed

$\rho(0, t)=a(t)$

as well as the initial condition

$$
\rho(x, 0)=\rho_{0}(x)
$$

least during a short period of time. In the study it is assumed that both functions $a(t)$ and $\rho_{0}(x)$ are continuous in some vicinity of 0 so that the behaviour of these two functions in the vicinity of origin is similar to the one in the origin itself. First of all, as known from section 2, only $a(t) \leq \rho_{c}$ can be considered. Secondly, as learned from the Riemann problem (16)-(17), if the value on the boundary is to be imposed, (i. e. the first alternative from the BLN condition (12) is to happen), we need to suppose that

$\rho_{0}(0)<a^{*}(0)$

where $a^{*}(t)>\rho_{c}$ is defined from $a(t)$ in a way that $q(a(t))=q(a *(t))$.

Condition (20) is the compatibility condition between the initial and the boundary value that must 
hold if the Dirichiet boundary condition (19) is to be imposed, at least for a while. Of course, far from the origin the initial condition can take a value larger than $a^{*}$ causing a shock that, after some time interval $\left[0, \mathrm{t}^{*}[\right.$ (that can be very short or infinitely long) reaches the boundary $x=0$, and forces one to choose the other alternative from (12), i. e. to impose only $\rho(0, t)>a *(t)$. It means that, for $t>t *$ the boundary condition (19) is actually no longer valid, as depicted below. The same effect can be produced, not only by growing initial density but also by the other boundary condition at $x=$ $l$.

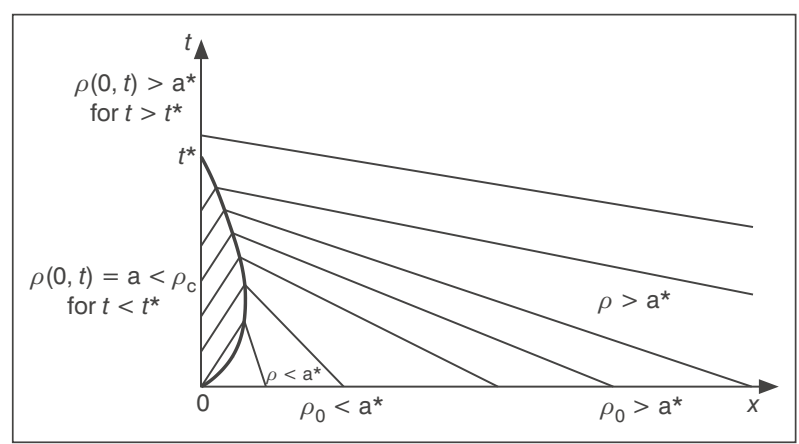

Figure 8 - Shock hits the boundary at time $t=t^{\star}$ and changes the equality $\rho(0, t)=a(t)$ to inequality $\rho(0, t)>a^{*}(t)$ on the boundary $x=0$

Similar analysis can be done for the right boundary condition

$$
\rho(l, t)=b(t)
$$

leading to the compatibility condition

$\rho_{0}(l)>b^{*}(0)$.

\section{FURTHER DISCUSSION OF BLN CONDITIONS FOR LWR MODEL}

The key argument that will be used to explain the BLN boundary conditions in real life is that high traffic density (at least in the LWR model) at some point of road is always caused by the traffic ahead of that point, while low density can only be consequence of the traffic behind the point in consideration. Let this phenomenon be analyzed in more details.

\subsection{The entry}

At point $x=0$ (the entry), according to [2], the value of density $\rho$ cannot be prescribed if that value is larger than $\rho_{c}$. Why is that so? Because it is not possible to prescribe the precise boundary value that will lead to heavy traffic. Indeed, as noticed in Remark 2, the value $a(t)$ can be seen as $\rho(0-, t)$, i. e. as the value of the density for $x<0$. Since heavy traffic at point $x=0$ is, in general, caused by the traffic conditions ahead of it (e. g. congested road), the high entry den- sity cannot be created only by a huge inflow independently of the conditions ahead. For instance, if the density for $x<0$ is as high as possible ${ }^{2}$ (i. e. $\left.\rho_{\max }\right)$ and the road is empty (or almost empty) in front of $x=0$ (i. e. $\rho$ is small for $x>0$ ), there will be the maximal flow, i. e. the optimal density at $x=0$, corresponding to the boundary condition

$$
\rho(0, t)=\rho_{c} .
$$

On the contrary, if the initial density $\rho_{0}$ satisfies the compatibility condition (20), the value $a(t) \leq \rho_{c}$, can be prescribed at least for a small interval of time, because light traffic at point $x=0$ can be caused by a small inflow. Obviously, if there are not enough cars entering the road that would lead to small density on the observed part of the road (unless the traffic on it was initially very heavy in the sense that the compatibility condition was violated). As in Example 1 and Figure 2 high density at $x=l$ can create a shock that reaches the boundary $x=0$ at some time $t^{*}$ and changes the boundary condition to $\rho(0, t) \geq a^{*}(t)$. The same kind of effect can be caused by the initial density. If the solution does not develop shocks, then the value of the density $a$ remains prescribed. If $a>\rho_{c}$, as noticed before, prescribing the exact heavy traffic density would mean to presume the density in the part $\langle 0, l\rangle$ (possibly) contradicting the conservation law. We can only prescribe that $\rho(0, t)>\rho_{c}$.

Of course, if the initial density was high near the entry $x=0$, and high means higher than $a *(t)$, then the boundary condition at $x=0$ has no influence on the solution regardless of its value.

\subsection{The exit}

The situation at the exit $x=l$ is the opposite. Indeed, one can prescribe high density at the end of the road (assuming that the initial density satisfies the compatibility condition (21)). An example of situation that would cause heavy traffic at point $x=l$ is the red traffic light at $x=l$. In that case there would be $\rho(l, t)$ $=\rho_{\text {max }}$. Of course, if low density is prescribed at the entry and high at the exit, then there is necessarily the shock wave. Unlike the previous case, the exact low density at the exit cannot be prescribed. That seems to be in consistency with real life. Indeed, there is no device that can be placed at the exit to guarantee the prescribed low density. Low density at $x=l$ can only be created by light traffic regime at the considered inter$\mathrm{val}\langle 0, l\rangle$ which is governed by the equation and it cannot be prescribed.

Finally, the meaning of the compatibility conditions (20) and (21) can be commented. What they mean is rather simple. Low density at the entry of the road cannot be prescribed if the initial density was high. The same goes for the exit, where high density cannot be imposed if the initial density was low. But 
(20) and (21) tell us more than that. They actually yield formulas to compute the precise values that guarantee the balance between the initial and the boundary condition and make the model feasible.

\section{BOUNDARY CONDITIONS ON THE FLOW}

It should be emphasized that the relation between $\rho$ and $q$ is not a one-to-one ${ }^{3}$ relation. Indeed, each value of $q \in\left[0, q\left(\rho_{c}\right)\right)$ is assumed by two densities $\rho$ and $\rho^{*}$, one lower and the other higher than $\rho_{c}$. Therefore, prescribing the flow is not equivalent to prescribing the density. Using that fact one can prescribe the flow at the point $x=0$ by prescribing the appropriate density $\rho_{\text {entry }}(t) \leq \rho_{c}$. Let $q_{\text {entry }}(t) \in\left[0, q\left(\rho_{c}\right)\right]$. We choose $\rho_{\text {entry }}(t) \leq \rho_{c}$ such that $q\left(\rho_{\text {entry }}(t)\right)=q_{\text {entry }}(t)$. Then the condition

$\rho(0, t)=\rho_{\text {entry }}(t)$

implies the condition

$q(0, t)=q_{\text {entry }}(t)$

in the BLN sense. In case of boundary condition for flow the BLN version of the condition (23) in fact means that

$q(0, t) \leq q_{\text {entry }}(t)$.

Due to the choice of $\rho_{\text {entry }}$ the equation (4) for $t>0$ and $x>0$, with initial condition (5) and boundary condition (22), understood in the BLN sense, (i. e. the semi-infinite highway problem) has a unique entropy solution. Furthermore, that solution satisfies the inflow condition (24) at the entry of the highway $x=0$ only in the BLN sense. Indeed, the initial value $\rho_{0}$ can be chosen such that the shock develops and reaches boundary $x=0$ at some instant $t^{*}$, after which the boundary value can no longer be prescribed (as depicted in Figure 2). At least for a while. During that period its upper bound can be merely prescribed, as written in (24).

The same thing could be tried with the two-point boundary value problem (i. e. with the three-detector problem). In that case for the given $q_{\text {entry }}, q_{\text {exit }}$ the $\rho_{\text {entry }} \leq \rho_{c}$ and $\rho_{\text {exit }} \geq \rho_{c}$ would have to be chosen such that

$$
q\left(\rho_{\text {entry }}\right)=q_{\text {entry }}, q\left(\rho_{\text {exit }}\right)=q_{\text {exit }}
$$

The exit condition, understood in the BLN sense, reduces to the inequality

$q(l, t) \leq q_{\text {exit }}(t)$

Then the problem (4), (5) with boundary conditions

$$
\rho(0, t)=\rho_{\text {entry }}(t), \rho(l, t)=\rho_{\text {exit }}(t)
$$

in the BLN sense (i. e. with conditions (24) and (25)), has a unique entropy solution. However, (unless $q_{\text {entry }}$ $=q_{\text {exit }}=\rho_{c}$ ) there is $q_{\text {entry }}<q_{\text {exit }}$ and such a solution will necessarily develop shock waves that would, eventually, hit the boundary at some time $t^{*}$. After that time one can only impose the upper bound for the density on the boundary. Therefore, the LWR problem with an inflow and an outflow condition

$$
q(0, t)=q_{\text {entry }}, q(l, t)=q_{\text {exit }}
$$

in the BLN sense, has an entropy solution but it cannot be smooth unless both $q_{\text {entry }}$ and $q_{\text {exit }}$ are equal to the road capacity.

The problems arising in the implementation of a finite-difference scheme when the flow boundary condition is imposed for the LWR model (and also for some second order models), was considered before by H. M. Zhang in [14]. He studied the flux condition directly using the Lebacque's demand and supply functions ([8]) and then proposed a simpler approach to prescribe the flow by prescribing the density, as done here. He obtained the correct boundary condition by considering the Riemann problem approach of Dubois and Le Floche, in each time step. This approach is, of course, equivalent to the BLN conditions (9)-(10) but more suitable for numerical computations.

\section{CONCLUSION}

This paper analyzes some choices of physically relevant and mathematically feasible boundary conditions for the standard LWR model of the traffic flow. The Dirichiet's conditions, namely, for the density (or velocity) and for the flow, in case of the bounded part of the road $[0, l]$ or semi-infinite road $[0,+\infty)$ are discussed.

It has been shown that the value $a(t)$ of the vehicle density $\rho(x, t)$ can he prescribed at the entry $x=0$ only if it is smaller than the optimal density $\rho_{c}$. while the density at the exit $x=l$ can be prescribed only if it is larger than $\rho_{c}$. Furthermore, if the boundary conditions are to be imposed, the initial and the boundary densities $\rho_{0}(x), a(t)$ and $b(t)$ must satisfy the compatibility conditions (20) and (21).

Both boundary conditions can be prescribed only in the BLN sense (see (12)-(1.5)). It means that, in case the initial density $\rho_{0}(x)$ is not too large in the vicinity of the entry (not too small in the vicinity of the exit), one can prescribe the value of the density on the boundary $x=0(x=l)$ for some period of time but it remains prescribed only until the shock wave develops (if it develops) and reaches the boundary. After the shock hits the entry (the exit) one can only ask for the density $\rho$ to satisfy the inequalities

$$
\begin{gathered}
\rho(0, t)>\rho_{c} \text { plus } q(\rho(0, t)) \leq q(a(t)) \\
\left(\rho(l, t)<\rho_{c} \text { plus } q(\rho(l, t)) \leq q(b(t))\right) .
\end{gathered}
$$

Unlike the density, any physically reasonable value of the flow can be prescribed at the ends of the road, of 
course, only in the BLN sense. The BLN boundary condition for the flow means that the value of the flow is prescribed until the shock reaches the boundary and only the upper bounds for the entering and the exiting flow after the shock arrives.

An interpretation of BLN conditions in the context of traffic flow is given.

The results of this paper can be used for practical computations in real life. To simulate a situation on a crowded highway, with some actual data, one first needs to find out whether the LWR model can be applied. This can be found by verifying the compatibility conditions, that have been derived. If the LWR model is applicable, the numerical simulations can be performed. To do so it is necessary to find a way how to prescribe the boundary conditions at the ends of the road, i. e. how to impose the given values of the traffic density or the traffic flow on the boundary. This method is described here in detail and an interpretation is given for each step.

\section{SANJA MARUŠIĆ, Ph.D.}

University of Zagreb,

Faculty of Transport and Traffic Sciences

Vukelićeva 4, HR-10000 Zagreb, Republic of Croatia

\section{SAŽETAK}

U ovom radu proučavamo rubne uvjete za standardni LWR model za opisivanje prometnog toka. Općenito opisujemo rubne uvjete u BLN smislu (Bardos, Leroux i Nédélec). $U$ kontekstu prometnog toka BLN uvjeti imaju prirodnu interpretaciju. Razmatramo rubne uvjete za gustoću i za tok te njihovo značenje u stvarnoj situaciji.

\section{KLJUČNE RIJEČI}

LWR model, prometni tok, hiperbolički zakon sačuvanja, rubni uvjeti

\section{REFERENCES}

1 see [3] for precise defnition

2 such situation can be created by the red traffic light at the entry
3 except for the California model

\section{REFERENCES}

[1] Ansorge R.: (1990) What does the entropy solution mean in the traffic flow theory?. Transportation Research B 24 (2), 133-143.

[2] Bardos C., A. Y. Leroux A. Y., Nédélec J. C.: (1979) First order quasi linear equations with boundary conditions, Communications in Partial Differential Equations 4 (9), 1017-1034.

[3] Daganzo C. F.: (1996) Fundamentals of transportation and traffic operations, Pergamon, Amsterdam.

[4] Dubois F., Le Floch P.: (1988) Boundary conditions for nonlinear hyperbolic systems of conservation laws, Journal of Differential Equations 71, 93-122.

[5] Gisclon M.: (1996) Étude des conditions aux limites pour un systčme strictement hyperbolique, via l'approximation parabolique, Journal de Mathématiques Pures et Appliquées 75, 485-508.

[6] R. Haberman R.: (1998) Mechanical vibrations, population dynamics and traffic flow. SIAM, Philadelphia.

[7] Godlewski E., Raviart P. A.: (1996) Numerical approximation of hyperbolic systems of conservation laws, Springer Verlag, New York, Berlin, Heidelberg.

[8] Lebacque J. P.: (1996) The Godunov scheme and what it means for first order traffic flow models, In: Proceedings of the 13th International Symposium on Transportation and Traffic Theory.

[9] Lighthill M. J., Whitham J. B.: (1955) On kinematic waves. I: Flow movement in long rivers. II: A theory of traffic flow on long crowded roads, Proceedings of the Royal Society A 229, 281-345.

[10] Marušić S.: (2000) Fluid models in the traffic flow theory, Promet-Traffic-Traffico. Vol 12, No 1, 7-14.

[11] Richards P. I.: (1956) Shock waves on the highway, Operations Research 4. 42-51.

[12] Serre D.: (1996) Systèmes de lois de conservation, I, II, Diderot. Paris.

[13] Witham J. B.: (1974) Linear and nonlinear waves, Wiley, New York.

[14] Zhang H. M.: (2001) A finite difference approximation of a non-equilibrium traffic flow model, Transportation Research B, 35 (4), 337-365. 